\title{
A avaliação de intervenções sociais como potencial instrumento de construção do conhecimento
}

The evaluation of social intervention as a potential knowledge building tool

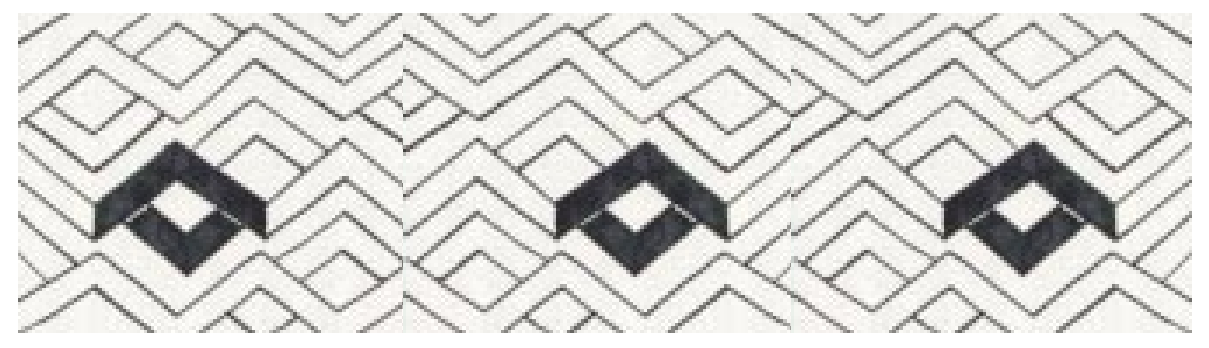

Auristela Maciel Lins ${ }^{1}$

\section{Introdução}

As avaliações de intervenções, sejam estas programas, projetos, atividades ou mesmo ações mais estruturadas, no campo da Saúde e da Educação se originaram do século passado e têm sido objeto da preocupação de pesquisadores dos diversos setores do conhecimento (estatísticos, economistas, educadores, epidemiólogos, cientistas sociais etc) de forma mais sistemática a partir da Segunda Grande Guerra, quando o Estado, obrigado a intervir mais eficientemente nas desigualdades sociais, passou a subvencionar uma série de projetos e programas de desenvolvimento das áreas sociais (Contandriopoulos et al., 1997; Lins, 1997). A avaliação se constituía, então, em parte do planejamento de programas e mantinha uma preocupação fundamentada na relação custo/benefício do mesmo. Os economistas foram por isso os grandes mestres na avaliação e trouxeram para o campo social as preocupações e a visão desenvolvidas junto ao setor produtivo industrial. Aos poucos, a incorporação de pesquisadores de outros setores e a insuficiência demonstrada pela abordagem economicista aplicada ao setor social, foram transformando a avaliação dessas intervenções em um objeto complexo e multidisciplinar. Hoje, a formulação de uma abordagem

\footnotetext{
1 Doutoranda do Departamento de Medicina Preventiva e Social da UNICAMP (Universidade Estadual de Campinas) e Consultora de Planejamento da Fundação Nacional de Saúde/MS. <auristela_lins@uol.com.br>
} 
de avaliação já se desprende do planejamento de programas e passa, em alguns casos, até a incorporá-lo, no sentido de que a avaliação de necessidades deve vir antes da decisão da formulação de um programa. Hoje, também, não mais se pensa na avaliação como uma atividade assistemática $e$ amadora. Principalmente as associações profissionais, criadas na América do Norte, reivindicam a atividade de avaliação de intervenções como uma especialidade e de competência de profissionais treinados.

Afora as disputas corporativas aí presentes, a complexidade dos objetos da avaliação de intervenções sociais tais como a Educação e a Saúde têm mesmo exigido uma ampliação das teorias e técnicas nas quais a avaliação tem se apoiado nessas últimas décadas. A importância e complexidade desses objetos têm promovido a necessidade da incorporação de diferentes técnicas, métodos e referenciais teóricos de inúmeras disciplinas vinculadas às ciências duras ou às Ciências Sociais, gerando diferentes abordagens metodológicas e diferentes disputas dentro da avaliação em geral, mas também promovendo a construção de novos conhecimentos para a melhoria das condições de vida dos indivíduos e da comunidade.

Nesse sentido, este trabalho tenta discutir a conformação da avaliação de intervenções como campo de convergência de diferentes disciplinas e sua contribuição para a construção do conhecimento no campo social, principalmente seu papel de instrumento multi, pluri ou interdisciplinar quando utilizado em programas que se propõem a atuar na interface Saúde/ Educação, mais especificamente na formação e educação continuada de profissionais da saúde como estratégia para a transformação da assistência à Saúde e da qualidade de vida de uma comunidade.

A avaliação de intervenções sociais como campo de convergência de disciplinas

A avaliação é um campo complexo. Esta afirmação possui diversos pressupostos, incorpora diversos momentos históricos e esconde inúmeros conflitos. Por isso também tem sido difícil encontrar definições $e$ classificações mais consensuais. Uma das definições mais aceitas é a de Scriven, que diz que "avaliar é julgar o valor ou mérito de qualquer coisa" (Worthen et al., 1997; Lins, 1997; Stenzel, 1996), muito longe, porém, de ser consensual.

Outra vertente da complexidade da avaliação vem de sua construção enquanto instrumento auxiliar de intervenção social. Direta ou indiretamente, a avaliação, de forma mais estruturada enquanto avaliação de projetos ou programas, sempre esteve ligada a propostas do Estado ou da Sociedade Civil de intervenções sociais, seja no sentido de produzir e acumular mais valia, seja do ponto de vista da intervenção sobre as injustiças sociais. A avaliação fazia parte do plano de ação $e$ do planejamento mais amplo. Nesse sentido, seu grande crescimento no mundo ocidental após a Segunda Grande Guerra incorporou a imposição de mérito e valor impostos

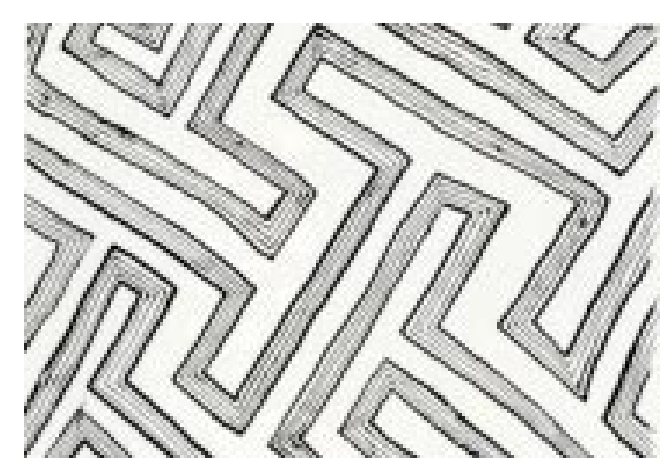


${ }^{2}$ Anotações de aulas da disciplina de pósgraduação em Saúde Coletiva da UNICAMPSP: História e Paradigmas do conhecimento em Saúde, ministrada pelo prof. Everardo Duarte Nunes. aos que se concentravam do lado ocidental, por meio da hegemonia capitalista, no desenvolvimento econômico, e do positivismo e neopositivismo, no desenvolvimento científico.

No entanto, a dinâmica das sociedades para as quais essas intervenções foram planejadas impôs limites à tentativa de desenvolvimento da avaliação enquanto instrumento quantitativo e objetivo e incorporou outras visões de mundo. Concepções estas mais amplas, que não colocavam as sociedades enquanto simples sistemas gradualmente adaptáveis. A avaliação foi, portanto, se ampliando além da perspectiva econômica e incorporando cada vez mais as disciplinas das Ciências Sociais e Humanas e com isto teve também de incorporar a complexidade de seu "novo" objeto.

A avaliação de intervenções ditas sociais, como Saúde, Educação, desenvolvimento de grupos específicos (adolescentes, diferentes grupos étnicos, religiosos, portadores de patologias específicas) e outros, no meu entender, passou a se caracterizar como um campo de convergência de disciplinas, individualizou-se e assumiu sua autonomia com relação ao planejamento e a outros tipos de avaliação ultrapassando, assim, segundo Foucault, seu limiar de positividade (Nunes, 1998²). Essa convergência de disciplinas para o campo da avaliação de intervenções sociais levanta a discussão sobre o caráter multi, pluri ou interdisciplinar da avaliação.

$\mathrm{Na}$ tentativa de analisar a construção do conhecimento e de propor intervenções no campo social que ultrapassem os limites impostos pelas disciplinas, vários autores têm discutido o caráter dessas construções e intervenções do ponto de vista das relações que se estabelecem entre as disciplinas dentro de um campo de conhecimento (Japiassu, 1976; Minayo, 1994; Almeida Filho, 1997) e, mais especificamente, na avaliação de intervenções (Yin, 1994; Hartz \& Camacho, 1996; Contandriopoulos et al., 1997). Japiassu (1976) faz uma distinção entre multi, pluri, inter e transdisciplinaridade, classificando-as com relação à negação e superação ou não das fronteiras disciplinares e propõe o interdisciplinar como o remédio mais adequado à cancerização ou à patologia geral do saber, ressaltando porém não considerá-lo como a panacéia científica. Minayo (1994) ressalta alguns conceitos e fundamentos presentes na obra de Japiassu (1976), incorpora outros pensadores que trataram do tema e alerta para o risco de se estar tratando a interdisciplinaridade como funcionalidade da ciência.

É por não concordar com a utilização indiscriminada do conceito e para precaver-me contra o uso funcional do mesmo, como pode-se depreender de modismos periódicos; por estar tentando compreender as relações entre as

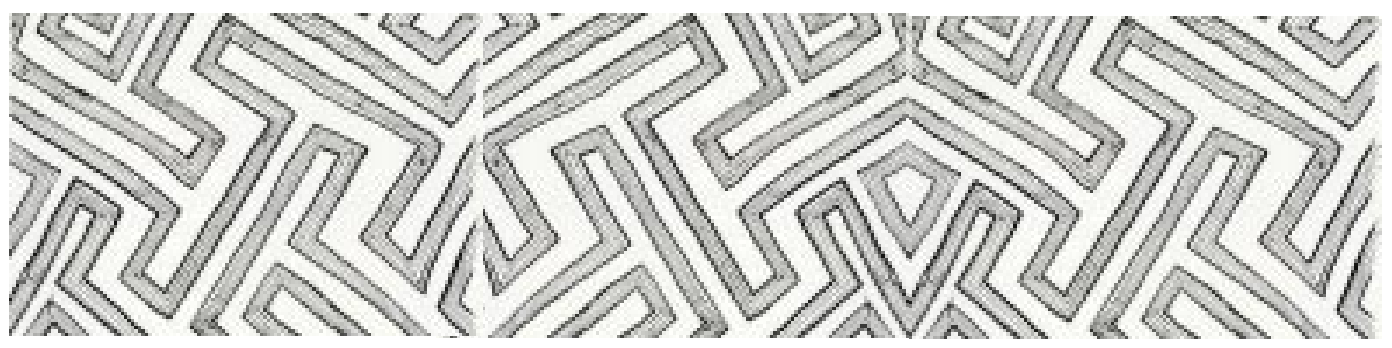

fevereiro, 2001 
diversas disciplinas no campo da avaliação de intervenções sociais e por concordar com Castoriadis (apud Minayo, 1994) que lembra que os problemas do conhecimento estão profundamente vinculados à organização $e$ orientação histórica da sociedade e não podem ser resolvidos sem uma profunda transformação das mesmas, que tenho aqui me utilizado da expressão "convergência de disciplinas" para caracterizá-la.

As contribuições da avaliação de intervenções sociais na construção do conhecimento

Entre os autores que defendem um tratamento à avaliação de intervenções com status de pesquisa científica, Contandriopoulos (1997) classifica a avaliação como "normativa", quando ela resulta da aplicação de critérios e normas e como "pesquisa avaliativa" quando elaborada a partir de um procedimento científico.

Considerar a avaliação de intervenções sociais como pesquisa científica é mais ou menos tranqüilo entre os autores, porém o dissenso se dá de maneira acentuada no trato de seu caráter. O leque vai desde considerá-la uma coletadora de dados para analisar o grau de eficácia de uma atividade, como definida por Suchman (Stenzel, 1996) a considerá-la um potencial instrumento de fortalecimento de grupos e indivíduos participantes de uma organização ou programa. Mesmo instalado, o dissenso não evita que a maioria dos autores e práticos da avaliação tratem-na como uma pesquisa que aplica conhecimentos já existentes e não com a possibilidade de que ela possa contribuir para a construção de novos conhecimentos.

Aplicar conhecimentos de forma responsável e competente já é uma forma de contribuir com o avanço do setor social, porém defendo que, enquanto tratada como pesquisa de segunda mão, a avaliação de intervenções sociais não vai conseguir dar conta da complexidade de seu objeto.

Discutindo a crise na Saúde Pública, seus sinais, explicações e propostas de abordagens para a mudança, Tarride (1998) defende a explicação da crise como uma questão epistemológica, no sentido que devemos buscar enfoques mais abrangentes para a compreensão da realidade, pois sua crítica à Saúde Pública, tanto sob a ótica dos referenciais teóricos, quanto sob a ótica da prática, não consegue se desvincular do Modelo Médico Hegemônico. Ele lança mão de Menéndez para definir o que entende por Modelo Médico Hegemônico: "o conjunto de práticas, saberes e teorias geradas pelo desenvolvimento do que se conhece como medicina científica". Nessa tentativa de ampliar referenciais e métodos, o mesmo autor, citando Nájera, afirma que "a questão não é incorporar as ciências sociais ao pensamento sobre saúde, e sim tratar a saúde como questão social" (Tarride, 1998, p.32) e propõe incorporar o adjetivo "complexa" à Saúde Pública, como caminho de mudança dirigido a sua forma de "olhar" seu objeto e a seu "proceder".

São vários os autores, no campo da Saúde Coletiva e da Educação, que têm trazido a "Teoria do pensamento complexo", formulada por Edgar Morin, para tentar compreender a crise e as saídas, principalmente

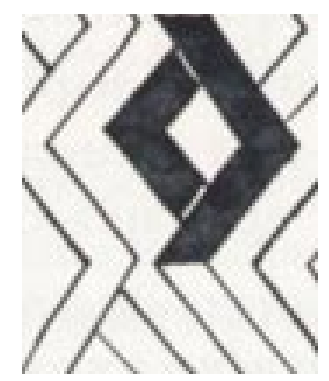


discutindo a questão da inter $e$ transdisciplinaridade para a compreensão teórica dos fatores intervenientes e para a formulação das abordagens de intervenção sobre

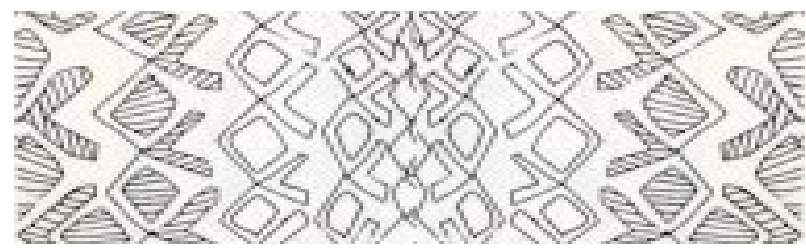
a crise nesses campos (Almeida Filho, 1997; Tarride,1998).

Fazendo uma crítica ao método científico que privilegia a análise das partes na compreensão do todo, mas não promove a síntese integradora $e$, conseqüentemente, criticando o pensamento simplificador, Morin (pud Tarride, 1998) desenvolveu seu pensamento complexificador ou complexo em que, partindo do paradigma sistêmico, defende o reconhecimento e o enfrentamento da complexidade presente tanto no objeto, quanto no observador e no modelo construído para observar o fenômeno.

Morin afirma que no mundo da organização vivente estão inerentemente presentes equilíbrios, acordos, concorrências, antagonismos e conflitos e as contradições presentes não são problemas insolúveis, e sim progresso do conhecimento (Tarride, 1998). Por isso o desafio do pensamento complexo é o de tratar a unidade/falta de unidade da vida sem reduzir, capturar ou debilitar um dos termos. As idéias contrárias devem ser pensadas em conjunto e sem incoerências. Para isso ele propõe o encontro de um metaponto de vista que relativize a contradição e a inscrição em uma circularidade, que torne produtiva a associação das noções antagônicas complementares. O paradoxo, o círculo vicioso e a antinomia seriam, portanto, fontes geradoras de pensamento complexo (Tarride, 1998).

Dessa forma, aos objetos de interesse das ciências, em especial das Ciências Humanas e Sociais, tem sido cada vez mais atribuído o adjetivo "complexo". Almeida Filho (1997), definindo o objeto complexo como sintético, não linear, múltiplo, plural e emergente, defende que para uma abordagem do mesmo, a organização da ciência em disciplinas autônomas e estanques deve ser superada pelas formas de interação entre elas.

Defendendo também que os espaços institucionais $e$ as disciplinas são permanentemente ocupadas por sujeitos da ciência, agentes históricos da prática científica, formula a idéia de "operadores transdiciplinares da ciência”. Esses agentes históricos são caracterizados pelo autor como "mutantes metodológicos, capazes de trans-passar fronteiras, à vontade nos diferentes campos de transformação, agentes transformadores $e$ transformantes" (Almeida Filho, 1997, p.18).

Para objetos complexos, portanto, pensamento complexo. Sendo a Saúde e a Educação objetos complexos, avaliá-los passa a requerer referenciais, técnica e métodos que possam se aproximar dessa complexidade, das suas antinomias e paradoxos. Assim, a avaliação de intervenções sociais, para onde convergem disciplinas, deve se manter aberta às novas aquisições, mesmo correndo o risco de que o que se acredita novo seja apenas travestido. Deve, principalmente, atuar no binômio ação-reflexão com a perspectiva não apenas de pensar indicadores, instrumentos e criar técnicas, mas de construir métodos que possibilitem o desvendar de novos conhecimentos.

Em pesquisa anterior (Lins, 1997) observei que a efetividade ou não de 
intervenções sociais está estreitamente vinculada a diversos fatores como a coerência de sua formulação; a potência do paradigma explicativo diante da complexidade do objeto; a construção dialética dos determinantes e condicionantes que se dá na relação sujeito-objeto e a utilização de instrumentos que possam captar e fazer aflorar a complexidade do objeto $e$ da relação que se estabelece com o observador /sujeito.

Observo, aqui, que a avaliação tem potencialidades para ser este instrumento e mais ainda, um instrumento de construção de conhecimentos, mas a tarefa não é simples. Acredito que ela deverá ser reconstruída e se autoreconstruir nesse processo, porém pensar a complexidade da tarefa, do objeto, da relação e pensá-la como campo aberto à convergência de disciplinas e como passagem dos que se propõem como "operadores transdicisplinares” pode ser o início da investigação para o como fazer.

\section{Referências bibliográficas}

ALMEIDA FILHO, N. Transdiciplinaridade e Saúde Coletiva. Ciênc. Saúde Col., v.2, p.5-19, 1997.

CONTANDRIOPOULOS, A. P., CHAMPAGNE, F., DENIS, J. L., PINEAULT, R. A avaliação na área da saúde: conceitos e métodos. In: HARTZ, Z. M. A. (Org.) Avaliação em saúde: dos modelos conceituais à prática na análise da implantação de programas. Rio de Janeiro: Fiocruz, 1997. p.2947.

HARTZ, Z. M. A., CAMACHO, L. A. B. Formação de recursos humanos em epidemiologia e avaliação dos programas de saúde. Cad. Saúde Pública, v.12 (supl.2), p.13-20, 1996. JAPIASSU, H. Interdisciplinaridade e patologia do saber. Rio de Janeiro: Imago, 1976.

LINS, A. M. O Programa UNI da Fundação Kellogg no Brasil: uma avaliação a partir do postulado de coerência de Mario Testa. Campinas, 1997. Dissertação (Mestrado) Faculdade de Ciências Médicas, Universidade Estadual de Campinas.

MINAYO, M. C. S. Interdisciplinaridade: funcionalidade ou utopia? Saúde e Sociedade, v.3, p.42-64, 1994.

TARRIDE, M. I. Saúde Pública: uma complexidade anunciada. Rio de Janeiro: Fiocruz, 1998.

STENZEL, A. C. A temática da avaliação no campo da saúde coletiva: uma bibliografia comentada. Campinas, 1996. Dissertação (Mestrado) Faculdade de Ciências Médicas, Universidade Estadual de Campinas.

YIN, R K. Discovering the future of case study method in evaluation research. Eval. Pract., v.15, p.28390, 1994

WORTHEN, B., SANDERS, J., FITZPATRICH, J. Program evaluation, alternative approaches and pratical guidelines. California: Sage Publications, 1994.

PALAVRAS-CHAVE: Conhecimento; estudos de avaliação; Educação em Saúde. KEY WORDS: Knowledge; evaluation studies; healthcare education. PALABRAS-CLAVE: Conocimiento; estudios de evaluación; Education en Salud.

Recebido para publicação em 24/08/00. Aprovado para publicação em 28/11/00.

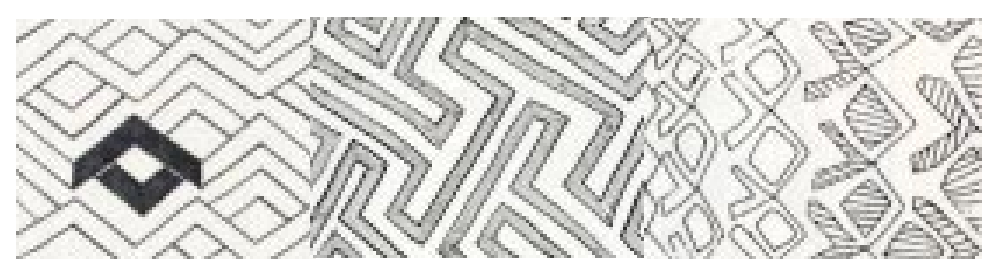

